Shenglan Zhang*

\title{
A systematic review of pedagogical research on teaching Chinese as a foreign language in the United States - from 1960 to 2020
}

\author{
https://doi.org/10.1515/caslar-2021-2003
}

\begin{abstract}
Since the 1960s, research on Chinese language teaching and learning has developed gradually, so it is worthwhile to synthesize the research results and examine trends and changes. A literature review shows that research has been done to synthesize research findings regarding Chinese language acquisition, but no review of the research studies have been done examining Chinese language pedagogy. This study investigates the research trends in Chinese language pedagogy in the U.S. by analyzing 122 empirical studies published in 25 peer-reviewed journals. These articles were identified through an exhaustive search using multiple databases and methods. The articles were cross analyzed in terms of research topics, language learning settings, methodological approaches, theoretical perspectives, and types of learners that were studied. The analysis found that the topics studied in pedagogical research have become increasingly diversified, and that many of the studies used SLA theories, cognitive theories, and multimedia theories as support for their research designs. Almost half of the studies used technology as part of their pedagogical method. The data also revealed that more research was done with beginning-level learners than advanced learners, and that most of the studies used qualitative data. Analysis of the data highlights the need for more research using Action Research and Design-Based Research methods and reveals a need for all future research to use theoretical support for the research designs. This article concludes by suggesting aspects of Chinese pedagogy that could benefit from further research.
\end{abstract}

Keywords: pedagogical research; research methods; research trends; theoretical perspective; use of technology

摘要: 自上世纪六十年代以来美国的中文教学研究在教与学两方面并行日益成 熟, 其结论值得总结。文献显示学者对习得方面的研究有过综述, 但是对教学 法方面的综述研究仍然缺乏。过去六十年中文教学方法的研究有什么趋势, 这 些研究集中在教学中的什么问题上, 所采用的研究方法以及涉及的理论有哪

*Corresponding author: Shenglan Zhang, lowa State University, 3118 D Pearson Hall, Ames, IA 50011, USA, E-mail: shenglan@iastate.edu 
些? 这些问题迫切需要回答。这些问题的答案可以为学者、教师以及中文师资 培训指出一个发展方向。通过对相关学术期刊进行穷尽式搜索与综述, 本文试 图回答这些问题。穷尽式搜索得到一百二十二篇实证性研究, 从发表日期来 看, 研究数量成递增趋势, 研究领域也呈现多样化。多数研究运用定性数据, 并且针对初学者教学的研究数量多于中级和高级者。文章最后探讨了中文教学 法研究的欠缺方面 (比如行动研究与 '设计为本” 研究法的缺乏; 研究设计应该 以扎实的理论为基础），以及将来的可能走向。

关键词: 教学法研究; 研究趋势; 理论视角; 研究方法; 科技运用

\section{Introduction}

While the popularity of learning Chinese has waxed and waned in the United States over the years, the overall trend shows increased demand for Chinese language instruction, particularly in recent years. Consequently, programs for educating Chinese language teachers, which are usually situated in an East Asian Languages and Cultures program, have expanded to offer M.A. programs in order to produce a greater number of qualified Chinese teachers. To train these teachers effectively, M.A. programs should base instruction on both anecdotal teaching experience and research conducted in the Chinese teaching classroom. To that end, this study examines the research already done in Chinese language teaching and notes the aspects of Chinese teaching that still need research. This knowledge can provide focus and direction for future research.

Although American educational institutions have offered Chinese instruction since 1877, it was not until in the 1960s that a significant number of people (over 1,000 students in the entire country) started to learn Chinese (Yao and Yao 2010). While scholars have conducted research on specific aspects of the history of Chinese language teaching and learning in the U.S. (Chi 1989; Meng 2017; Yao and Yao 2010; Zhang 2018), no studies examine the development of Chinese pedagogy research in America.

The field of Second Language Acquisition (SLA) bifurcated into basic SLA research (aka, pure research) and applied SLA (aka Instructed SLA) over time (Han 2016). Pedagogy falls within the Instructed SLA area of research, as the academic discipline of pedagogy refers to the theory and practice of teaching. As noted by McLaren and Jaramillo (2007), the discipline studies teaching methods to determine which are most effective at imparting knowledge and skills efficiently in an educational setting. Pedagogy involves setting educational goals, identifying methods to help reach those goals, creating learning environments that facilitate those methods, and observing interactions in which the goals may be achieved. 
The importance of Chinese pedagogy in improving learning outcomes was first recognized in print in 1979 (Zhang 2018) when the editor of the flagship journal of Chinese Language Teachers Association (CLTA), Professor Light, debuted a column in the journal to address pedagogy. However, it was in 1989 that Chi pointed out that Teaching-Chinese-as-a-Foreign-Language (TCFL) was not involved in research on language pedagogy. He wrote,

\footnotetext{
In the literature and at conferences, there is extensive discussion on issues relating to linguistics analysis of the language or to the merits and weaknesses of certain texts used in the classroom. However, on the whole, there is not enough discussion on language pedagogy at either the theoretical or practical level. Most of us seem to be either unaware of or uninterested in the research activities, past and present, in the fields of language acquisition and second language teaching, and in the Shingling theoretical extensions and practical implications such research may have for teaching Chinese as a foreign language (p. 113).
}

As we strive for high-quality, research-based classroom instruction (Wen 2019), it is important to learn what pedagogical research has been done and what still needs to be done. The purpose of this study is to investigate the development of the pedagogical research in the field of TCFL in the United States from 1960 to the present.

\section{Literature review}

In previous studies, researchers have devoted a great deal of attention to the acquisition of Chinese as a second or as a foreign language in the United States (e.g. Ke and Li 2011; Ke and Shen 2003) and in China (e.g. Shi and Wen 2009). Ke and Shen (2003) did a review of literature study on Chinese-as-a-Foreign-Language (CFL) acquisition in America starting from the early 1980s to 2003. They analyzed the research done on the acquisition of Chinese as a foreign language during those 20 years by examining a few aspects of language learning, including the acquisition of pronunciation, characters, grammar, and reading strategies that learners adopted; non-cognitive factors that affects learning; learners' pragmatic knowledge; and assessment. Their study also analyzed the pedagogical research on teaching writing/composition. At the end of the article, the authors offered valuable suggestions on the gaps in the field of CFL acquisition research.

Ke and Li (2011) detailed the situation in teaching Chinese nine years ago, giving an overview of programmatic structures, opportunities, and challenges in the field. Their study also surveyed research on learning Chinese as a foreign language from the late 1980s to the first decade of the 21st century. Although their study focused on students' learning rather than pedagogy, it offered a clear picture 
of the research situation in the field of CFL. In addition, Ke and Li (2011) gave insight on what issues required more attention to improve our understanding of CFL learning, such as calling for more CALL research and more CFL researchers.

Other researchers have approached Chinese language teaching from other perspectives. For example, Meng (2017) analyzed Chinese language teaching in the context of the changing language policy patterns that America showed from 1876 to 2016. Meng divided the 130-year history into four periods based on the policy patterns and Chinese teaching, such as the establishment of Chinese language programs and the enrollment numbers corresponding to the different policy patterns.

Taking a different perspective, Yao and Yao (2010) gave a detailed description of the start of Chinese language teaching in the United States. They analyzed the development of Chinese language teaching in the mainstream and nonmainstream society in the 19th century, the 20th century, and the current time, and pictured the future development of Chinese teaching in America.

Zhang (2018) gave an interesting report of the growth of the journal Chinese as a Second Language (CSL, formerly titled Journal of CLTA) between the years of 1966 and 2018. The article covers many areas, ranging from the CLTA membership to the role of linguistics and literature in the research published in CSL. Because CSL is the flagship journal of CLTA in America, this narrative, to a large degree, mirrors the growth of CSL in America. It stressed the "unwavering commitment to pedagogy" of the journal; however, noted that there was no space provided in the journal to talk further and in depth about the pedagogy research.

In another research project, H. Zhang (2014) and Z-S. Zhang (2014) provided statistics on different analyses of articles published in Journal of CLTA (JCLTA), evaluating them in groupings such as language, type, and category and by theme in the title. The article offers a valuable overview of the publications, the trends, and the blind spots in JCLTA from 1966 to 2014. As H. Zhang (2014) and Z-S. Zhang (2014) mentioned, however, the methodology of this study might be too simplistic, JCLTA may not reflect the entire field of CFL, and more details and analyses are needed to provide a more comprehensive and in-depth account of the publications in JCLTA.

All these studies have made significant contributions to the fields of CSL and CFL. They provide a useful account of the beginning of CSL/CFL in the U.S. and its growth over the years as well as research on how students acquire Chinese as a foreign or a second language, which is the basis for designing instructional approaches for classroom use. After discovering how learners acquire or learn the language, it is crucial to then discover how to teach the language based on the findings about students' methods of learning. Learning and teaching are two separate matters, even though they are closely connected. No studies in the 
literature have systematically examined the development of pedagogical research - that is, research on the instructional approaches used to teach Chinese as a foreign language or as a second language - in CFL from the infancy of field to the present. Few efforts have been made to investigate whether the development of pedagogical research in teaching the Chinese language has a solid theoretical basis. A review of the literature focusing on the development of CFL pedagogy in the U.S. using a careful search criterion and a rigorous research method is urgently needed.

\section{Research questions}

This study seeks to answer the following questions:

1) What are the trends in Chinese pedagogical research in the United States from 1960 to the present?

2) What pedagogical research topics have attracted the most attention from scholars and what topics have been neglected in the past six decades?

3) What research methods have been adopted in these pedagogical research studies and what theories underpin these studies?

\section{Methodology}

To answer the research questions, three search methods were used to conduct an exhaustive search identifying all the peer-reviewed publications related to Chinese pedagogy research studies in the United States published in the time period from 1960 through mid-2020 when this project was conducted.

The first search method was a database search. Four databases that include all the important journals in the field were used for the search: Linguistics and Language Behavior Abstracts (LLBA), ERIC, PsychInfo, and Education Index Retrospective. The databases complement each other, as LLBA includes journals in the field of linguistics and languages, Eric includes journals in the field of education in general, PsychInfo includes journals in educational psychology, and Education Index Retrospective includes journals that address all levels of education and education specialties. When searching these databases, "Chinese" was used as the keyword to include all possible articles, and searches were made using a variety of different settings to be sure to gather all relevant results. A manual search was then done to screen the titles and gather relevant articles.

The second search method was a manual search of the references found in the articles gathered through the initial search. The purpose of the manual search was 
to find relevant articles that the initial search of the databases might have missed. The third search method, which was the most time-consuming but most productive method, was a manual search of the two most important journals in the field of CFL in America: Chinese as a Second Language (i.e. Journal of the Chinese Language Teachers Association), the flagship journal of Chinese Language Teachers Association, and Journal of Technology and Chinese Language Teaching (JTCLT). These two journals were indexed at a later date than the more inclusive journals such as Foreign Language Annals (FLA); therefore, some issues of the journals might not have been indexed in the databases at the time of the initial search. A manual search of these two journals ensured that the search included all published articles in the field of Chinese pedagogy. To ensure that the search was exhaustive, the author also visited the website of each journal included in the databases used for search method \#1 and used the search archive function on each journal website to manually search all the relevant journals.

The search used the following criteria to gather the sample: 1) The study had to include empirical research methods. In other words, each study had to create knowledge from actual experience through observation and measured phenomena, and the study had to include a description of the methods used to examine a certain population or phenomena including the data selection criteria and the instrument(s) used. Concept papers, reports of teaching experiences, narratives about specific innovations in the classroom, and teachers' reflections on or description of lessons, though valuable in many aspects in the field, were not included in this study. 2) The research topic had to be Chinese pedagogy; that is, the topic needed to be a method or methods of teaching modern Chinese as a foreign or a second language. Note, if a study addressed culture or film as part of the language teaching, it was included. If the study focused exclusively on culture or film, it was not included. An example of such a study that was excluded is $\mathrm{Mu}$ and Zhang (2005), which studied the use of WebCT to teach Chinese films. It is true that research findings in many other areas found their way to helping with Chinese teaching over time, such as acquisition of Chinese as a second language, Chinese learning motivation, teacher education, assessment, learner behaviors such as the use of reading strategies, study abroad, teaching Chinese literature or film, the acquisition of a certain language phenomenon, and so on. However, because they are not directly related to methods of teaching Chinese, articles focused on these topics were not included. 3) The study had to be conducted in the United States. Because the focus of this study is pedagogical research in America, studies that were published in America but conducted in another country were not included.4) The research had to be published in a peer-reviewed journal between January 1960 and June 2020. 5) The articles had to be written either in English or in Chinese. 
The searches using these three search methods resulted in a set of 898 articles that were related to Chinese language learning and teaching. These 898 articles were further screened manually based on the above criteria, and 122 articles were identified as the pool of articles that satisfied all of the criteria. Information about each of the 122 articles was recorded, including the year of publication, the research setting (k-12 schools or college/university), the research topic, learner information (proficiency level, heritage or non-heritage), the research method, and the theoretical framework. This information was then categorized based on the aspects of language use that the research focused on, such as characters, vocabulary, reading, grammar, pragmatics, listening, speaking, essay writing, pronunciation, culture, or overall language proficiency. The data was then analyzed.

\section{Findings and discussion}

\subsection{Journals that published Chinese pedagogical research}

The finding shows that 25 peer-reviewed journals published Chinese pedagogical research in the United States in the past 60 years. The three journals that published the most articles related to Chinese pedagogical research are Chinese as a Second Language (CSL), Journal of Technology and Chinese Language Teaching (JTCLT), and Foreign Language Annals (FLA). Seven journals published five or more studies in the area of pedagogy, and 15 journals published one to four relevant studies in the past six decades. See Figure 1. Note: the number of publications in each journal does not necessarily reflect the emphasis of the journal because the inauguration date of each journal is different. For information on the different inauguration

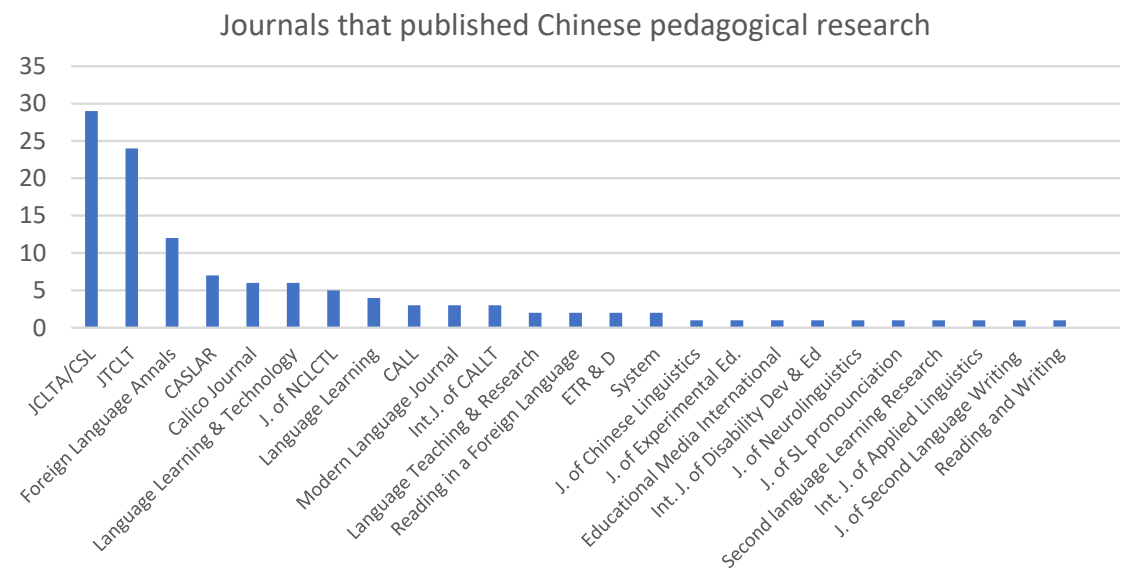

Figure 1: Twenty-five journals published Chinese pedagogical research. 
dates of the few journals in America that focus on Chinese languages, please see $\mathrm{H}$. Zhang (2014) and Z-S. Zhang (2014).

\subsection{Trend in time of publication and the use of technology}

Out of the 122 studies, only three ( $2 \%$ ) were published before the late 1980 s and $98 \%$ of the 122 articles were published after revolutionary theoretical and teaching changes happened in the 1980s. The two biggest changes in Chinese pedagogy during that time were: 1) Communicative learning started to be widely accepted in the field, which revolutionized L2 classroom teaching; 2) Instruction started to change from formfocused (such as audio-lingual instruction and habit formation) to meaning focused. However, the findings show that not all studies published after this time concentrated exclusively on meaning-focused teaching. There was some renewed interest in formal instruction in the context of meaning-based communicative methods because formal instruction, especially in teaching complicated grammars, could improve accuracy and prevent fossilization (Yuan and Dietrich 2004).

Another trend relates to the number of studies that addressed the use of technology in teaching Chinese. There were 60 studies that utilized computer and internet technology representing nearly $50 \%$ of the studies in total. This is a continuation of a trend in CALL research detected nine years ago (Ke and Li 2011) and this theme was discussed in Da and Zheng (2018). Most of these articles were also published after the 1980s. See Figure 2.

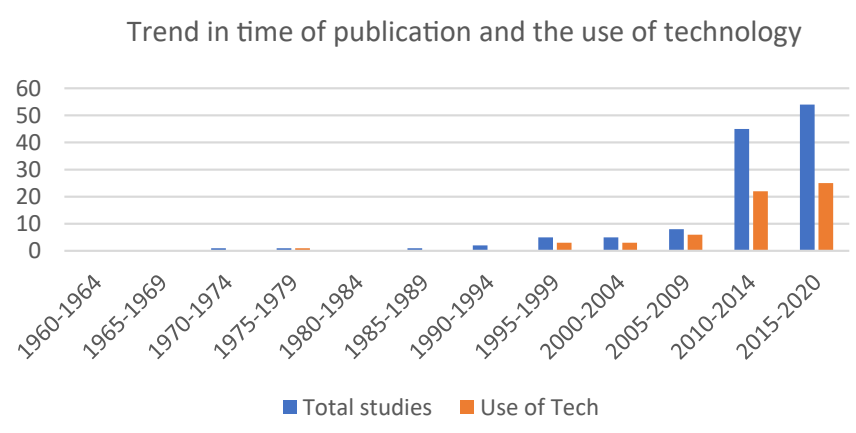

Figure 2: Time distribution of the publications and studies regarding the use of technology in teaching.

\subsection{Research settings and the level of learners in the research}

Out of the 122 studies, 105 studies (86\%) were conducted in a university setting, and the learners were largely college students. Twelve of the 122 studies 
(10\%) focused on teaching Chinese to middle school and high school students. Five (4\%) focused on teaching Chinese to kindergarten and elementary school students. Out of the five studies that were conducted in an elementary school, four of them were about teaching Chinese in an immersion program. See Figure 3.

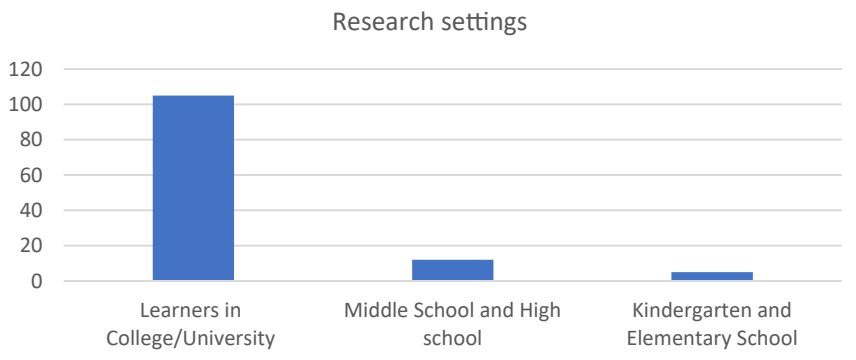

Figure 3: Research settings: college/university, middle/high school, or kindergarten/ elementary school.

Out of the 105 studies investigating university students, six focused on teaching heritage learners and three focused on teaching in an intensive program. Some of the 105 studies examined students at the same language level and some examined students at all language proficiency levels (beginning, intermediate, and advanced). Two studies did not specify the language mastery levels of the students who participated in the studies.

Of the 105 studies, 61 (58\%) targeted beginning-level CFL learners, 43 (41\%) targeted intermediate-level CFL learners, and 24 (23\%) targeted advanced-level learners (See Figure 4). While most did not sub-categorize within each level, some studies made the distinction between intermediate-low, intermediate-mid, and intermediate-high; for the purpose of maintaining simplicity in this study, the sublevel within intermediate level students was not recorded. When identifying advanced level students, all learners who had studied more than three years in a college setting were categorized as advanced level.

College CFL learners' language level in the pedagogical studies

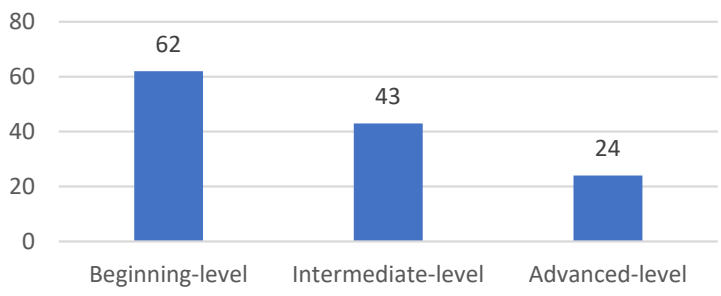

Figure 4: Learners' language level in the pedagogical studies in the college/university setting. 


\subsection{Research method adopted in the pedagogical studies}

A majority of the studies (46\%) used qualitative data gathered through various data collection methods such as surveys, case descriptions, discourse analyses, exploratory methods, comparative analyses and so on. About $36 \%$ of the studies were experimental studies, out of which only two were longitudinal studies. Eighteen percent of these studies adopted mixed methods, combining both qualitative and quantitative methods within the same study. See Figure 5.



Figure 5: Research methods adopted in the 122 studies.

\subsection{Aspects of language teaching addressed}

The 122 pedagogical studies were sorted into two general categories. Category 1 includes studies that focused on evaluating the overall effect of a particular pedagogical approach at the syllabus or program level. Category 1 includes 31 articles, or $25 \%$ of the studies. The overall effect being examined in these studies included aspects of learning such as self-efficacy, communication skills, verbal skills, cognitive awareness of the language, collaboration, engagement, enjoyment, and classroom management. Category 2 includes studies that focused on one aspect of language teaching. There were 91 articles (75\%) that fell into this category. Research topics addressed in these studies included 10 aspects of the Chinese language: characters (e.g. Jin 2006*; Wang 2014*; Xu, Perfetti, and Chang

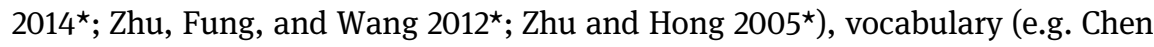
2016*; Li and Tong 2019*; Yang and Xie 2013*; Shen 2010*; Shen and Xu 2015*),

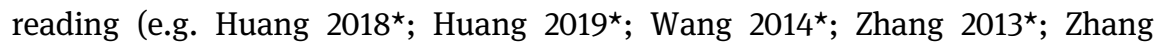
2016b*), grammar (e.g. Paul and Grüter 2016*; Yuan 2012*; Zhang 2018*; Zhang $2017^{\star}$ ), pragmatic use of the language (e.g. Li and Taguchi $2014^{\star}$ ), pronunciation (e.g. Chun $2015^{\star}$ ) and tones (e.g. Godfroid, Lin, and Gyu 2017*), culture (e.g. Wang and Crooks 2015*; Zhang 2019a*), writing (Eubanks, Yeh, and Tseng 2018*; Liao 2018*; Yuan 2010^), listening (e.g. Wilberschield and Berman 2004*), and speaking (e.g. Stickler and Shi 2013^). (The citations marked with an asterisk ${ }^{\star}{ }^{\star}$ can be found in the overall references of the included studies on CASLAR website.) Note: Because a few studies addressed both reading comprehension and learning vocabulary and one study focused on both reading and writing, the total number of the studies in Figure 6 exceeds 91. 


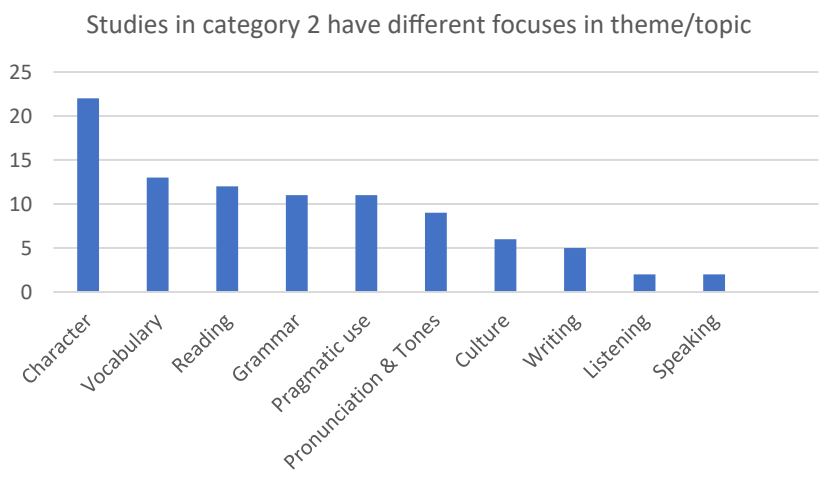

Figure 6: The different focuses among the category 2 studies.

\subsubsection{Studies in category 1}

The 30 studies in category 1 have the same distribution trend as the group of 122 studies; that is, there were few publications of this type before the 1980s, and it was not until after the year 2000 that the majority of the studies were published. The trends in learners' heritage or non-heritage status, in research settings, and in the type of research methods used in the studies mirrored those of the entire group. That is, a majority of the 30 studies in category 1 researched non-heritage learners in a college or university setting. There were more studies that used qualitative data than used quantitative data, and the fewest number of studies used mixed methods. However, the learner skill level studied did not follow the overall trend. More studies in this category worked with advanced level learners compared to the number of studies that worked with learners at the intermediate level. See Figures 7 and 8. Another difference is that there was a higher proportion of qualitative to quantitative studies in this group than in the overall group. See Figure 9.

The studies in category 1 covered a variety of instructional approaches, including the manner of teaching, e.g. co-teaching (e.g. Wang, Zhang, Liu, and Yonke 2019^); high pressure versus low pressure (e.g. Packard 1989^); the use of Chinese or English as the instructional language (e.g. Yang 2010*); the way to provide corrective feedback (e.g. Li 2014); the creation of different learning environments like blended learning (e.g. Zhang 2016a*), flipped classrooms (e.g. Tseng, Broadstock, and Chen 2016*; Yang, Yin, and Wang 2018*), wiki-enhanced Course Management Systems (e.g. Zhang 2019^), WebCT (e.g. Cheng 2010^), Virtual Reality (e.g. Chen 2011*; Grant and Huang 2010*), or One-on-One video conferencing (e.g. Sun and Chen $2017^{\star}$ ); the application of digital tools such as Google maps, WordPress, Adobe Spark (e.g. Valdebenito and Chen 2019^), or 
Category 1: Distribution over the years of thirty pedagogical studies looking into learners' overall changes

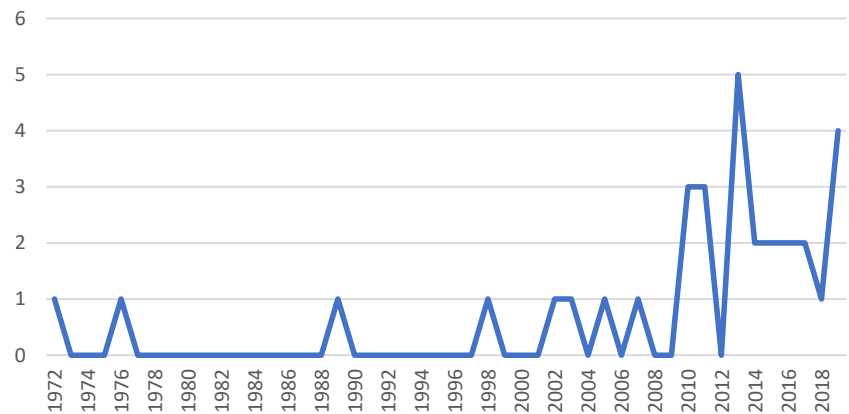

Figure 7: The publication time distribution of the category 1 studies.

Learner information in the category 1 studies

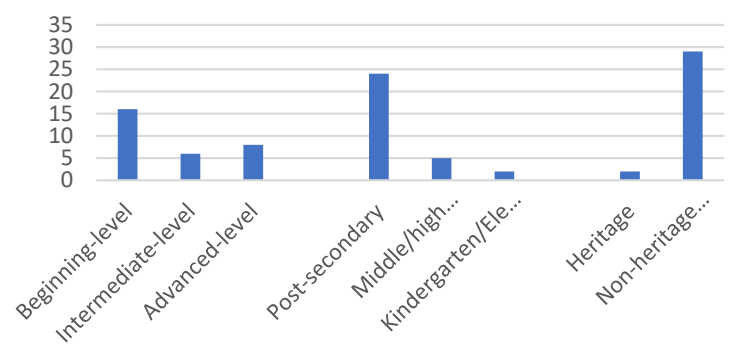

Figure 8: Learner information in the category 1 studies.

A comparison of methods: The category 1 studies and the 122 studies

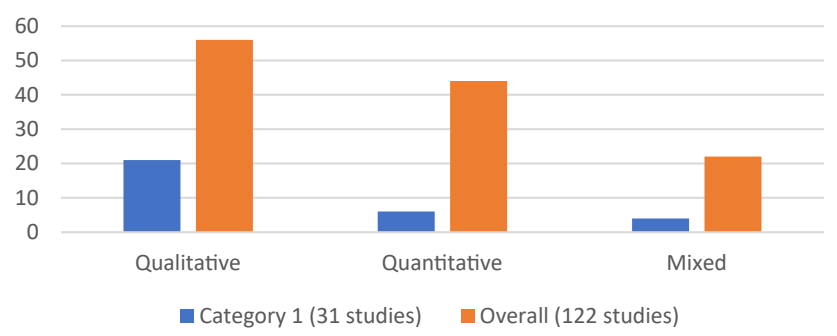

Figure 9: Research methods adopted in category 1 studies as compared to the 122 studies. 
speech recognition in class (e.g. Da 2015*); gamification (e.g. Tang and Taguchi 2019^; Wang, Liu, and Zhang 2019^); the use of different instructional approaches such as project-based and theme-based learning (e.g. Padilla, Fan, Xu, and Silva 2013*; Zhang and Beckett 2014^); the use of researcher-designed software or apps such as CyberChinese (e.g. Yu and Michael 1998*); the use of authentic multimedia

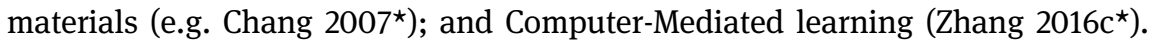
(The citations marked with an * can be found in the references page of the included studies on the CASLAR website.)

A majority of these studies (67\%) did not have a theoretical framework, while $23 \%$ of the studies relied on various theories to underpin their studies. Constructivism and meaningful learning were the most commonly used theories, and 50\% of the studies that had a theoretical framework were based on the Vygotsky's sociocultural and constructivist theories. These educational theories emphasize the importance of social participation, relationships between novices and experts, the setting of an activity, and how knowledge is constructed when learners interact with others in the real world. The Zone of Proximal Development and scaffolding were often referred to in the studies that used these theories. Theories used in the other half of the studies included Interaction Hypothesis (Long 1996), Noticing Hypothesis (Schmidt 1990), Output Hypothesis (Schmidt 1995, 2001), and the notion of intersubjectivity (Heritage 1984). See Figure 10.

Theories adopted to support the studies in category 1

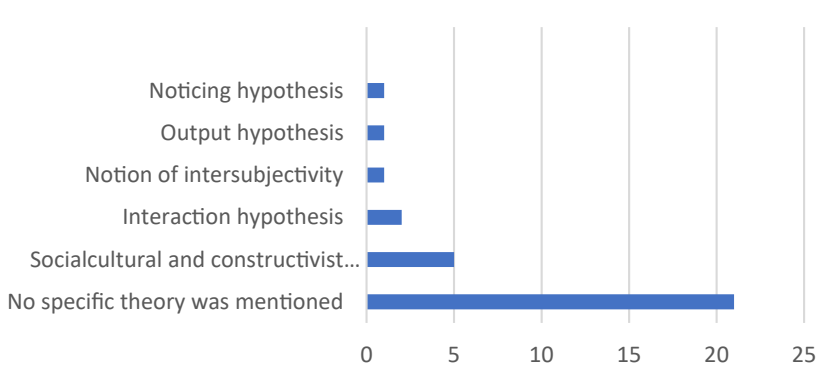

Figure 10: Theories based on which the studies were conducted.

\subsubsection{Studies in category 2}

Similar to the studies in category 1, the studies in category 2 (focusing on a specific aspect of Chinese language learning) also reflected a sharp increase in studies after the 1980s, in particular during the 21st century. Still, pedagogical research in Chinese character learning and grammar has a long history, beginning in the 
1970s. Pronunciation/tones and reading studies also have a relatively long span of research history compared to other aspects of teaching and learning Chinese. Chinese writing and the pragmatic use of the language was not studied often in earlier decades, but research in those areas has increased over the past 10 years. In fact, in the past decade, researchers increased the number of investigations into all aspects of Chinese language teaching. See Figure 11.

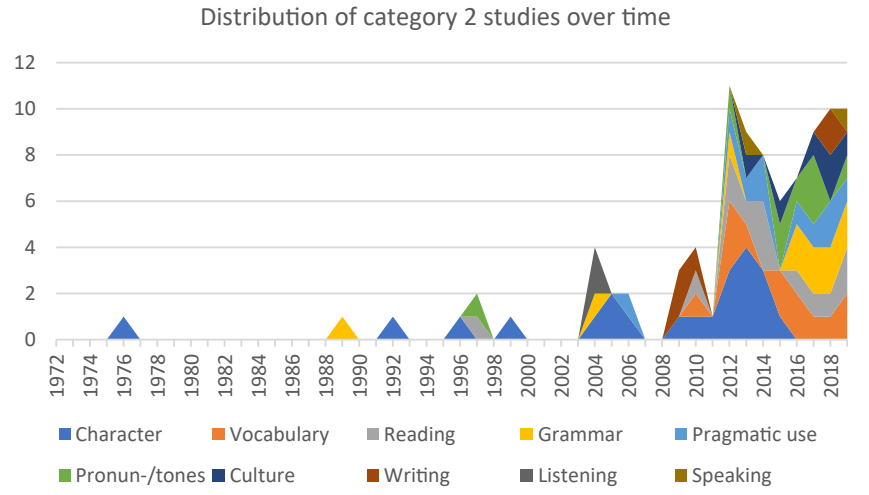

Figure 11: Distribution of pedagogical research in category 2 over time.

In the most researched areas of Chinese teaching and learning - such as characters, vocabulary, reading and grammar - a majority of the studies adopted an experimental design. In other research areas, most studies used qualitative data. Some of the studies in the most often researched areas also used mixed methods, although the studies using mixed methods represent a small percentage of the total studies. See Figure 12.

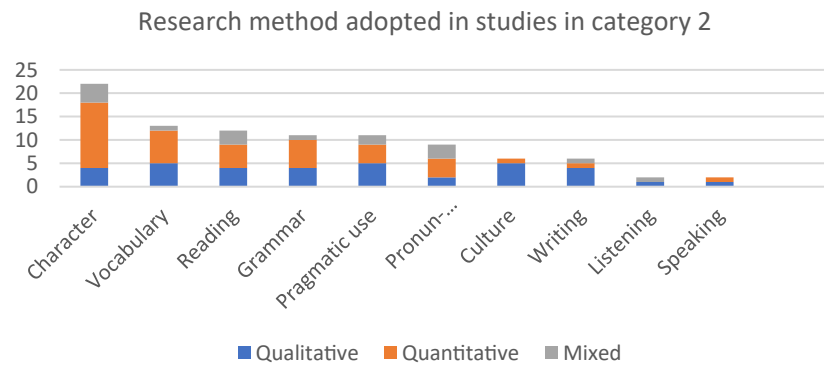

Figure 12: Research methods adopted in category 2 studies. 
In areas of Chinese learning that tend to be challenging for beginners - such as learning characters, pronunciation and tones, listening, reading, and grammar - a majority of the studies were conducted with learners at the beginning level, whereas in other areas - such teaching learners to use the language pragmatically - studies focused on advanced-level learners. Out of the 22 studies focused on teaching characters, four examined students who did not have any Chinese learning background. These four studies were included in the pool of studies examining beginning-level learners. In the studies that focused on teaching vocabulary, most focused on teaching intermediate-level learners. See Figure 13.

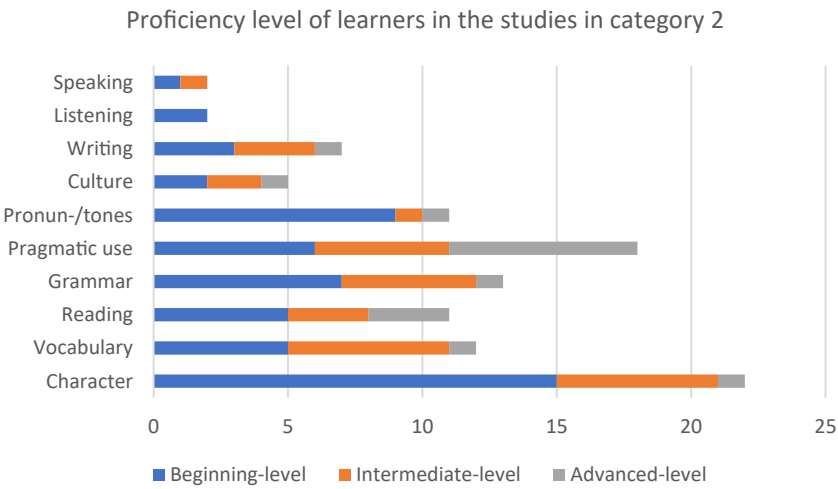

Figure 13: Proficiency level of learners in category 2 studies.

It is not surprising to find that heritage learners were studied in each of the three primary areas of teaching Chinese - reading, vocabulary, and writing. Most heritage learners do not have a problem speaking and listening, and most of them learned some writing as they grew up. Additionally, they are familiar with Chinese culture. The areas that heritage learners need to improve the most are essay writing, reading, and vocabulary. See Figure 14.

While most studies were conducted in a college/university setting, a few studies were completed in K-12 schools. For example, two studies on teaching characters were completed in a kindergarten or an elementary school, and a few of the studies on reading, vocabulary, writing, and listening were done in a middle school or a high school. See Figure 15.

\subsubsection{Studies on teaching characters}

Out of the 22 studies on teaching characters, 17 studies (77\%) used a theory to support their research, while five studies (23\%) did not. Among the theories 
Heritage or non-heritage: Learners in the studies in category 2

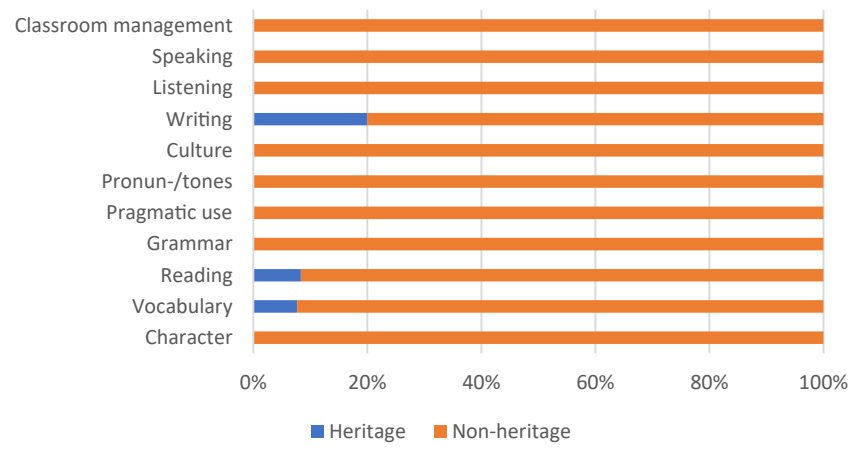

Figure 14: Heritage or non-heritage: learner info in the category 2 studies.



Figure 15: Learning settings in the category 2 studies.

underpinning these studies, Dual Coding Theory (DCT) (Paivio 1971, 1986) was the mostly widely used. According to Paivio's $(1971,1986)$ DCT theory, both visual and verbal information are used to represent information, but they are processed differently in distinct channels in the human mind. Therefore, information can be retained more successfully when the stored information is represented both verbally and visually than when represented with one or the other alone. Using DCT, many studies on teaching characters used experimental design to test the effectiveness of a variety of presentation methods. Other theories used in the studies are related to DCT including Cognitive Load Theory (Sweller 1988), Split-Attention Effect (Chandler and Sweller 1992), Cognitive Theory of Multimedia Learning (Mayer 2005, 2009), and Generative Theory of Multimedia Learning - a combination of Wittrock (1974)'s 
Generative Theory of Learning and Paivio's DCT. In addition, the Interactionist Approach (Chapelle 1998, 2009; Gass and Madden 1985) was also adopted in one of the studies. Another theory that was used to support the design of one study was the Order Analysis method, a multi-dimensional scaling technique that generates hierarchical sets of data points based on the relationships between the elements studied (Krus 1977), depending on which characters were ordered hierarchically rather than randomly in the study.

The primary focus in studies on teaching characters was on using different ways to present characters such as digital flashcards, characters in hierarchical order, different groupings of characters, static versus dynamic presentation, chunking mnemonics, embodied animation, translation, self-generated mnemonics, and a variety of multimedia presentation methods (digitally voiced pronunciation, stroke sequence animation, radical presentation, stroke order presentation, pinyin presentation, hyper-character, and so on). In addition, there was research on how to bypass teaching character writing by using a penless approach and research on when is the best time to introduce character writing to learners.

\subsubsection{Studies on teaching vocabulary}

Nine of the 13 studies on teaching vocabulary had a theoretical framework, while four (31\%) did not. The nine theory-based studies were grounded in the following theories: the Dual Coding Theory (Paivia 1971, 1986), the Cognitive Load Theory (Sweller 1988), the Generative Theory of Learning (Wittrock 1974), and the Cognitive Theory of Multimedia Learning (Mayer 2005, 2009). Additional concepts and frameworks were used in the studies to help develop methods and understand results. For example, the Active Learning Concept (Meyers and Jones 1993) derived from the Constructivist Learning Theory, Cooperative Learning (Johnson and Johnson 1975) derived from the Sociocultural theory, Motivational Dynamics (Waninge et al. 2014), and Memory-Based Strategic Framework (Ma 2014).

Studies defined Active Learning as learners being given the opportunity to participate in problem solving activities. Cooperative Learning in a classroom setting was defined in studies as organizing activities into academic and social learning experiences so that learners could capitalize on one another's resources and skills to complete tasks collectively as they worked toward academic goals. In the Motivational Dynamics framework, motivation was seen as dynamic and changeable in the process of language learning, and the framework asserted that teachers should direct the motivational current to help learners to benefit more from learning. Lastly, the Memory-Based Strategic Framework proposed that there are four stages of processing vocabulary learning, which go from perceiving the 
form to accessing the meaning to mentally building a new word and then to retrieving the new word from a mental lexicon for production.

There were diverse themes that were presented in studies about teaching vocabulary, and these themes were all associated with providing needed support for the learners to retain the new words better. These supports include, e-flashcards, e-dictionary, external supply of text-vocalization, and gamification, and different visual-verbal presentations. There were also studies on how to provide an internet retrieval tool to help students to learn collocation, how to use an iPad to assist learners in learning idioms, and how to use mobile technology to help learners learn colloquialism.

\subsubsection{Studies on teaching reading}

Eight out of the 12 studies on teaching reading did not have a theoretical framework. Four of the studies used theories and concepts as such LaBerge and Samuels's (1974) theory of automatic information processing in reading, Paivia's (1971) Dual Coding Theory, the concept of reading for deriving meaning and the social nature of reading (Farnham 1887), and the concept of linguistic input modification (Yano et al. 1994). Automatic information processing refers to a mental cognitive process that goes through a series of stages involving visual, phonological, and episodic memory systems to achieve comprehension. Automaticity happens as the result of repetitive training in this process. The concept of linguistic input modification says that both the simplification and elaboration of the input have value in helping learners with their reading.

The themes of these studies included providing different supports for helping readers' understanding, such as pop-up dictionaries or e-dictionaries, externally supplied text vocalization, visual aids, and multimedia aids. In addition, these studies used other methods such as repeated reading, the spiral model for performance, using authentic materials, using student-created picture books as reading materials, and using theme-based and content-based instruction.

\subsubsection{Studies on teaching grammar}

Seven out of the 11 studies on teaching grammar did not use a theory for support. Four studies adopted one of a handful of different theories, and some studies adopted more than one theory for their theoretical framework. This group of studies had the widest variety of theories adopted compared to studies addressing other aspects of the Chinese language. The theories that were adopted include the Teachability Hypothesis (Pienemann 1989), the assumption of language acquisition order and sequence (Hyltenstam 1977; Krashen 1985), the consciousness- 
raising theory (R. Ellis 2002; Ellis 2007), the level of processing framework from psychology (Craik and Lockhart 1972), the assumption of blocking (Ellis 2006; Kruschke and Blair 2000), the Full Transfer/Full Access hypothesis (FT/FA) (Schwartz and Sprouse 1996), the frequency effect hypothesis (N. C. Ellis 2002), the noticing hypothesis (Schmidt 1990), and the Cognitive theory of Multimedia Learning (Mayer 2005, 2009).

The Teachability Hypothesis is based on the idea that instruction is more effective when it is compatible with the development of the learner's interlanguage. It predicts that some grammatical structures can be best learned only when the learners are at the developmental stage to learn it. The acquisition order refers to the idea that one particular grammar feature must be acquired before others. The acquisition sequence refers to the process through which learners generally go when they learn a linguistic form; for example, the acquisition of a grammatical feature sometimes occurs in a U-shaped course rather than a lineal upward motion.

Consciousness raising involves increasing learners' awareness of certain linguistic features. It theorizes that learners have a long-lasting awareness of form and an improved accuracy when linguistic features are explicitly introduced. It is important to design consciousness-raising tasks when teaching challenging grammar. The level of depth of processing refers to conceptual or semantic processing versus perceptual processing. The former is deep processing, and the latter is shallow processing. For learners to have deep processing, the learners need to process information semantically in association with similar information and actively construct relationships between the information being processed and the information associated with it.

The blocking phenomenon is the effect of learner attention. There are many situations where the blocking phenomenon shows up in language learning; for example, when the cues are redundant. The FT/FA, on the other hand, hypothesizes "that the initial state of L2 acquisition is the final state of L1 acquisition (Full Transfer) and that failure to assign a representation to input data will force subsequent restructurings, drawing from options of Universal Grammar (Full Access)" (Schwartz and Sprouse 1996: 40). The frequency effect hypothesis postulates that the frequencies of linguistic items to which learners are exposed have important facilitative effects in language acquisition, and the noticing hypothesis argues that input has no practical value and cannot become available for learners to effectively process unless it is noticed by the learners.

The topics in this group of studies focused on how to teach challenging linguistic features in Chinese, such as the bă-construction, jìu, bèi, le, classifiers, and the modal verbs. The researchers in this group explored different ways of teaching grammar (high-pressure vs. low-pressure), using formal instruction, using explicit 
negative evidence, using a Task-Based Language Learning approach, and using multimedia design to engage and inform students' grammar learning.

\subsubsection{Studies on teaching using the language pragmatically}

Six out of the 11 studies on pragmatic instruction did not use a theory to support their study. The five remaining studies adopted four different theories, including the Principles of Training for raising learners' communicative strategies (Oxford 1994; Rabab'ah 2002), the Input Processing model (VanPatten 2004), the Skill Acquisition theory (Anderson 1993; DeKeyser 2001), and the Noticing Hypothesis (Schmidt 1990). The Skill Acquisition theory states that the different types of knowledge (mainly, declarative knowledge and procedural knowledge) are acquired first and then the automatization process happens. It explains language learning as a gradual transition from effortful use to more automatic use of the language and the ultimate goal is to achieve speed and accuracy in processing. The Input Processing theory explains how learners process input, how they make formmeaning connections, and how they map syntactic structures when speaking.

These studies addressed different approaches to teaching learners to make requests, to expressing gratitude, to refusals and complaints, to accepting invitations, to using formulaic expressions, to learning circumlocution, and to using modal verbs appropriately in contexts. Some of the studies used ComputerMediated Communication (CMC) or gamification to help learners to improve their pragmatic competence.

\subsubsection{Studies on teaching pronunciation and tones}

Out of the nine studies on teaching pronunciation and tones, a majority did not adopt any theories. One theory that was adopted among these studies was the Cognitive Load Theory (Sweller 1988). One of the studies focused on pinyin, applying speech recognition technology to find out if it helps with learners' pinyin accuracy. Eight studies focused on how to teach tones and three of them researched Tone3 production and/or perception. Five out of the eight studies investigated the effectiveness of different approaches to teaching learners tone production, and three studies addressed tone perception. The different approaches tested in these studies included displaying pitch contour to the learners, learners creating and comparing pitches to native speakers, comparing pitch heightfocused and pitch direction-focused instructions, using the multimodal method, comparing low teaching and full teaching approaches, the "Full T3 First" approach, and comparing online and traditional approaches to teaching tones. 


\subsubsection{Studies on teaching culture}

Most of the studies $(N=6)(83 \%)$ on teaching culture had a theoretical support. The theories used by the authors included the concept of Performing Culture (Walker 2000), the lens of internationalization (Lantolf 2000; Lantolf and Thorne 2007), Vygotsky's sociocultural theory, and the Cognitive Theory of Multimedia Learning (Mayer 2005, 2009). The concept of performing culture regards culture as what we do but not what we know. To understand another culture and another language, one must use the language and participate in the real life of the target culture. Using the lens of internalization, an individual's language learning process is seen as a reorganizing process that happens through collaborative work in a social environment and includes the steps of voluntary attention, logical memory, and formation of concepts. Internalization happens when the learner's reliance on external help decreases.

The themes used in this group of studies included the performed culture approach to teaching behavioral culture, allowing medical students to explore authentic doctor-patient conversations in order to improve their intercultural competence, using authentic videos to learn about social realities, combining researcher-edited multimedia and ethnographic interviews to teach students Chinese culture online, using an online cooperative learning project to increase learners' cultural awareness, and using decorative graphics in personalized instruction to teach culture.

\subsubsection{Studies on teaching writing}

Four of the five studies on teaching writing were supported by a theoretical framework. The theories used included the concept of classroom community (Potts 2005; Rovai 2001), Vygotsky's sociocultural and social constructivist theory, the assumption of benefits that computer-mediated writing instruction could bring such as de-centering authority (Phinney 1996), the theory of humans' limited attentional capacity (Anderson 1983, 1995), the concept of multiliteracies (The New London Group 2000), and the interactionist theory (Gass 1997; Long 1996; Swain 1995).

The concept of classroom community is crucially important, especially for virtual classrooms, because it offers learner interaction and shared knowledge construction while also providing for learner perception of the value of social bonds in the learning process. The concept of multiliteracies acknowledges the multiplicity of communication channels and linguistic diversity in the world today and has brought us a broader view of literacy than traditional views. The concept also provides an appropriate pedagogical framework for teaching literacy. The interactionist theory demonstrates that writing skills can be better acquired when writing activities are 
embedded in social dialogs. When learners are engaged in bi-directional output, they can test their output with each other, are able to notice the gaps in their learning, and are likely to attend to the problematic aspects of their interlanguage.

The themes in this group of studies included different approaches to improving writing (such as using WebCT discussion board), using different task performance conditions (form-focused vs. meaning-focused), conducting a technology integrated writing workshop (using an iPad recording app, iPad camera, and the Book Creator App), and using different forms of pre-task pair planning activities (face-to-face oral discussion vs. online text-chat).

\subsubsection{Studies on teaching listening and speaking}

One of the two studies on teaching listening did not have any theoretical support. The other study on listening did have a theoretical framework, as did both studies on teaching speaking. The theories used in the studies were Paivia's Dual Coding Theory, the socio-cultural perspective on language learning (Lantolf 2000; Vygotsky 1978), and the Educational Engineering Research Theory (Colpaert 2010; Colpaert 2016). The Educational Engineering Research Theory argues that neither technology nor pedagogy nor content should be the starting point for the design of a learning environment. To design a beneficial learning environment, real-world situations should be analyzed in a systematic and verifiable way and the design should take into account the specificity of the context.

One study on listening used WebCT-based, teacher-created listening materials to improving listening. The other study used video clips from authentic Chinese TV broadcasts with two different organizer conditions: one using words and sentences with pictures taken from the video and the other using words and sentences but without pictures. One of the studies on improving speaking focused on using synchronous online Chinese tutorials while the other used wiki-enhanced TBLT to improve learners' speaking.

\subsection{A summary of the major findings}

This study set out to answer three research questions: 1) What are the trends in Chinese pedagogical research in the United States from 1960 to the present? 2) What pedagogical research topics have attracted the most attention from scholars and what topics have been neglected in the past six decades? 3) What research methods have been adopted in these pedagogical research studies and what theories underpin these studies? 
The study found that trends in research include a sharp rise in the number of pedagogical studies conducted starting after the late 1980s. Technology is an important component of pedagogy studies, with about half of the studies using some type of technology. A little more than 4/5 of the studies were conducted in a university setting focusing on non-heritage learners, with far fewer studies focused on middle schools and elementary school. Out of the studies conducted at the university level, a little more than half targeted beginning-level CFL learners, and a little less than half targeted intermediate level learners, and only about $1 / 4$ targeted advanced-level learners.

When looking at topics, one quarter of the studies focused on evaluating the effect of certain pedagogical approach at the syllabus or program level addressing different aspects of learning such as self-efficacy, communication skills, cognitive awareness of the language, collaboration, engagement and so on. Three quarters of the studies addressed different aspects of language teaching. Ten aspects of the Chinese language were addressed including teaching characters, vocabulary, reading, grammar, pragmatic use of the language, pronunciation and tones, culture, writing, listening, and speaking, out of which, teaching characters, vocabulary, and reading were the top three most-researched areas.

Methods in the studies vary, with a little less than half of the studies using qualitative data only, less than 2/5 using experimental studies, and less than $1 / 10$ of the studies using mixed method. As many as $2 / 3$ of the studies lack a theoretical framework to underpin their studies. With the $1 / 3$ of the studies using theoretical support, the theory that was adopted most frequently was Constructivism, and a majority of the studies used Vygotsky's socio-cultural and constructivist theories. Other theories were also adopted, such as, the Long (1996)'s Interaction Hypothesis, Schmidt (1990)'s Noticing Hypothesis and Output Hypothesis, and the notion of Heritage (1984)'s intersubjectivity.

\section{Looking to the future}

As early as 2003, in Ke and Shen (2003)'s review of literature research on CSL acquisition, researchers noticed a limitation with the research in CSL; that is, the majority of studies focused on pronunciation, tones, and grammar. Ke and Shen called for diversifying research by including research on the other aspects of Chinese language teaching and learning, such as speaking, listening, reading, and writing. In the most recent two decades, pedagogical research in America is no longer focused solely on grammar, characters, pronunciation, and tones as it was in the first few decades of pedagogical research. The research in the past 20 years has addressed a variety of important pedagogical problems, including teaching 
speaking, listening, writing, the pragmatic use of the language, and understanding the Chinese culture. Research in all these areas should be continued. However, there are a few additional areas that should see more growth.

First, more pedagogical research on teaching in online and in flipped or blended environments is needed. While there has been a significant amount of research in the past two decades addressing the use of computer technology in teaching Chinese and there have been a few research studies on teaching Chinese in online, blended, and/or flipped situations, more research is needed, especially in the area of teaching in different environments. The ubiquity of the internet and the increase in online teaching in a variety of formats provides the opportunity for learners to learn on their own schedules and at their own paces. As a group, CSL educators should take advantage of this opportunity to find better approaches to making learning as flexible and as effective as possible. Furthermore, the pandemic has given most instructors the opportunity to teach fully or partially online. A majority of instructors now have first-hand online teaching experience. This has laid a foundation on which innovative and creative online instructional approaches can be built and tested.

These online teaching experiences will also affect how we utilize the affordances of technology when the time comes for returning to face-to-face instruction. It is crucial that instructors examine all aspects of online learning and study how to help learners receive the maximum benefits from these online learning environments. For example, there is a vast amount of free, engaging, multimedia material on the internet that can help learners understand culture and language, if it is used effectively. There is a great potential for using these resources to teach different levels of learners. The questions then arise: How should teachers integrate the available multimedia resources in teaching? If a teacher would like to design online components for learners, how should such a design be constructed? At a syllabus level, how should a blended course or a flipped classroom be built to enhance students' learning?

Second, more studies using the action research method are needed. The pedagogical research in the past six decades used a balanced variety of different research methods including experimental, exploratory, and some mixed methods. However, out of the 122 studies, there were only three studies using the action research method. This finding concurs with the findings in many studies, such as in Ke and Li's (2011) research, and supports other scholars' calls for more action research (McDonough 2006; Yuan 2018). Action research, a problem-focused research method, combines theory and practice via an iterative process (such as problem diagnosis, action intervention, and reflective learning) that involves both researchers and practitioners (or the practitioner as researcher) and provides teachers with structured reflection so they have a better understanding of 
themselves as teachers (Wallace 1998). It is a valuable research method that could contribute to professional growth as well as to the field of Chinese pedagogical research. Han (2016) agreed, calling for teachers to be aware that "the published studies were done in contexts different from the situations teachers find themselves in" and proposed that "teachers themselves should not be content with being consumers of research ... [but] be empirically minded, practicing so-called “empirical pedagogy" (Han and Selinker 1999: 247). Yang and Luo (2017) found that there was not a straightforward application of SLA to pedagogy, and they suggested that more language instructors should be engaged in pedagogical research. Furthermore, it has been suggested that even though there are effective strategies for teaching certain aspects of the language, it is still worthwhile for teachers to explore more effective strategies (H. Zhang 2014; Z-S. Zhang 2014; S. Zhang 2017). That said, further research is needed to reveal the reason(s) for the extremely small amount of action research published. If the problem is that Chinese language teachers lack training in this area, graduate-level Chinese language teacher education programs should start to emphasize this type of research.

Third, research needs to be based on theories and have an underpinning theoretical framework. This finding also concurs with Ke and Shen (2003) and their call for rigorous, theory-based research design. The findings of the current study show that about half of the studies utilized a variety of theories from SLA, especially from cognitive linguistics (based on cognitive science, ref. Jing-Schmidt and Peng 2018) and the sociocultural perspective, from theories in multimedia design. However, about half of the studies lack a theoretical framework to support the research design, the instructional tools, and the strategies. Theory is essential in any scholarly or scientific discipline. By framing the study with a theoretical foundation, the research results can be explained within an already established body of knowledge and the observed connections, giving credibility to the results and inspiring further research in areas in need of investigation (McMillan and Schumacher 2000). As stated in Klette (2011), "theories provide predictions and explanations as well as guidelines for actions and behavior” (p. 4). Without theories, studies lack a set of structured lenses through which the study can be designed, observed, investigated, and analyzed. In addition, theories provide protection against unscientific approaches to a problem, an issue, or a theme (Suppes 1974). By situating one's research within a theoretical framework, the research can be easily understood and protected against criticism. Not only should the research design be based on theories, but instructional tools, strategies, and approaches must also be based on an accepted theory of learning, cognition, or design (Bednar et al. 1995).

Fourth, more Design-Based Research (DBR) is needed. In some of the exploratory studies, there were detailed descriptions of how certain instruction or 
a particular instructional approach was designed. However, the designs did not employ specific theoretical claims about teaching and learning, nor did the studies draw conclusions about how the design could be improved and what principles could be drawn from the results to influence the next cycle of design. The value of DBR relies on developing solutions or innovations (i.e., interventions) to problems and then putting the interventions to use to find out how well they work and why. By so doing, DBR helps us understand the relationships among educational theories, designed solutions/approaches/innovations, and actual practice. In pedagogical research, most researchers are testing to discover the most effective instructional approach to help learners with a certain aspect of the language. It is important to know which approach has the most positive impact on learning. It is even more important to know why this approach has a positive impact. Only after we know the "why"- the principles of design and the theories on which the design is based - can practitioners and researchers design more successful approaches based on the principles and theories (Hampel 2006). The principles could include, for example, how to utilize the immediate learning environments and how to take into consideration the features of the learner group.

Fifth, even though the studies did touch on a large number of different topics and themes, there are still neglected but important areas where more research is needed. For example, there is only one study on classroom management in the 122 studies. Classroom management requires a wide variety of skills and techniques that instructors apply in either Face-to-Face meeting or online synchronous meetings in order to keep students organized, attentive, engaged, and academically productive. Strategies and approaches for teaching different aspects of the language are useful. However, without good classroom management strategies, it is hard to apply those teaching strategies and instructional approaches effectively. This research task is even more challenging now, because researchers not only need to discover the best classroom management practices for Face-to-Face meetings but also need to examine the management of online synchronous meetings and discover ways to better use online meeting time.

Another neglected area is that of teaching Chinese for specific purposes. Only two studies on teaching Chinese for a specific purpose were identified, one on teaching Chinese for business, one on teaching for medical purposes. Teaching Chinese for a specific purpose generally uses similar approaches as teaching the language in an academic setting; however, there may be specific strategies that best help the learners grasp skills in the specific contexts, whether it is in the area of business, medicine, engineering, agriculture, or other professions.

Sixth, research is needed to examine how to teach certain aspects of the Chinese language to the groups of learners that have not been thoroughly studied. In some research areas, such as teaching the pragmatic usage of the Chinese language, the 
published studies primarily focused on working with advanced-level learners. In other areas, such as teaching characters and pronunciation/tones, the studies published so far concentrated on beginning-level learners. It would be worthwhile to find different instructional approaches to teach tones and pronunciation in a remedial way to advanced-level learners and find ways to get learners to perceive their fossilized pronunciation and tones and produce them in the correct way. It is also possible and could be valuable to find ways to raise the pragmatic awareness of learners from early on in their Chinese language learning journey and teach beginning-level learners to use simple expressions pragmatically.

\section{Conclusion}

An exhaustive search of peer-reviewed journals resulted in 122 empirical studies on Chinese pedagogy in America published between 1960 through mid-2020. A look at the publication time and the theme of the studies found that there was an increased amount of pedagogical research on teaching Chinese as a foreign language over those decades. As many as 25 journals published pedagogical research in the past six decades. Over these years, the pedagogical research has become more diversified in terms of the issues in teaching being addressed.

This review study did not include narratives such as concept work and anecdotal accounts of teaching experiences (especially the large numbers of this type of work that were published in the 1960s, the 1970s, and in the early 1980s). For example, Nathan (1966) published an article "A classroom innovation: Contracts and Self-Paced Instruction in Chinese," which is a description of an innovation in classroom. However, the article did not investigate the effectiveness of the approach. Articles that utilized that kind of report format were not included in this review. However, those studies are the forerunners of the current research. Those researchers and practitioners did a great deal of exploration in teaching, especially in how to balance speaking, reading, listening, and writing. Examining those studies is a project worthy of another article or even a book to explore their impact. Additionally, another full article is warranted to describe the inception of the research in different areas of teaching Chinese as a Foreign language in the United States. Such an article would examine the issues in teaching and learning what concerned our forerunners, what they did to address those issues, and what we do to solve those problems now. Without the exploration and persistent efforts of those early researchers, current researchers could not have reached the level they have today. Their research is broader and considers many different factors, but is full of sincerity and devotion, which makes it valuable in many ways. 
Another limitation of this study is that there is not space to fully elaborate on the pedagogical studies in each aspect of language teaching. For example, there are 12 studies on teaching reading. A full article is needed in order to delve into each of the theories used, the trends shown across time, the themes addressed, and the future directions indicated. This holds true for most of the other studies that examine teaching the other aspects of language, for example, the use of technology, teaching characters, teaching cultures, and so on.

\section{References}

Anderson, John R. 1983. The architecture of cognition. Cambridge, MA: Harvard University Press. Anderson, John R. 1993. Rules of the mind. Hillsdale, NJ: Lawrence Erlbaum.

Anderson, John R. 1995. Cognitive psychology and its implications. 4th edn. New York: Freeman. Bednar, Anne, Donald Cunningham, Thomas Duffy \& David Perry. 1995. Theory in practice: How do we link? In Gary Anglin (ed.), Instructional technology: Past, present, and future, 2nd edn., 100-112. Englewood, CO: Libraries Unlimited.

Chandler, Paul \& John Sweller. 1992. The split-attention effect as a factor in the design of instruction. British Journal of Educational Psychology 62. 233-146.

Chapelle, Carol A. 1998. Multimedia CALL: Lessons to be learned from research on instructed SLA. Language, Learning and Technology 2(1). 22-34.

Chapelle, Carol A. 2009. The relationship between second language acquisition theory and computer assisted language learning. The Modern Language Journal 93(s1). 741-753.

Chi, Richard. 1989. Observations on the past, present, and future of teaching Mandarin Chinese as a foreign language. Journal of the Chinese Language Teachers Association 24(2). 109-122.

Colpaert, Jozef. 2010. Elicitation of language learners'personal goals as design concepts. Innovation in Language Learning and Teaching 4(3). 259-274.

Colpaert, Jozef. 2016. Big content in an educational engineering approach. Journal of Technology and Chinese Language Teaching 7(1). 1-14.

Craik, Fergus M. \& Robert S. Lockhart. 1972. Levels of processing: A framework for memory research. Journal of Verbal Learning and Verbal Behavior 11(6). 671-84.

$\mathrm{Da}$, Jun \& Yanqun Zheng. 2018. Technology and the teaching and learning of Chinese as a foreign language. In Chuanren Ke. (ed.),The Routledge handbook of Chinese second language acquisition, 432-447. New York: Routledge. https://doi.org/10.4324/9781315670706-20.

DeKeyser, Robert. 2001. Automaticity and automatization. In Peter Robinson (ed.), Cognition and second language instruction, 125-151. Cambridge: Cambridge University Press. https://doi. org/10.1017/cbo9781139524780.007.

Ellis, Nick C. 2002. Frequency effects in language acquisition: A review with implications for theories of implicit and explicit language acquisition. Studies in Second Language Acquisition 24(2). 143-188.

Ellis, Nick C. 2006. Selective attention and transfer phenomena in L2 acquisition: Contingency, cue competition, salience, interference, overshadowing, blocking, and perceptual learning. Applied Linguistics 27(2). 164-194.

Ellis, Nick C. 2007. The associative-cognitive CREED. In Bill VanPatten \& Jessica Williams (eds.), Theories in second language acquisition, 77-95. Mahwah, NJ: Lawrence Erlbaum. 
Ellis, Rod. 2002. Grammar teaching-practice or consciousness-raising? In Jack C. Richards \& Willy A. Renandya (eds.), Methodology in language teaching: An anthology of current practice, 167-174. Cambridge: Cambridge University Press. https://doi.org/10.1017/ cbo9780511667190.023.

Farnham, George L. 1887. The sentence method of teaching reading, writing, and spelling: $A$ manual for teachers. Syracuse, NY: C. W. Bardeen, Publishers.

Gass, Susan \& Carolyn Madden (eds.). 1985. Input in second language acquisition. Rowley, MA: Newbury House.

Gass, Susan M. 1997. Input, interaction, and the second language learner. Mahwah, NJ: Lawrence.

Hampel, Regine. 2006. Rethinking task design for the digital age: A framework for language teaching and learning in a synchronous online environment. ReCALL 18(1).105-121.

Han, Zhaohong \& Larry Selinker. 1999. Error resistance: Towards an empirical pedagogy. Language Teaching Research 3(3). 248-275.

Han, Zhaohong. 2016. Research meets practice: Holding off and holding on. Chinese as a Second Language 51(3). 236-251.

Heritage, John. 1984. Garfinkel and ethnomethodology. Cambridge: Polity Press.

Hyltenstam, Kenneth. 1977. Implicational patterns in interlanguage syntax variation. Language Learning 27(2). 383-411.

Jing-Schmidt, Zhuo \& Xinjia Peng. 2018. Linguistic theories and teaching Chinese as a second language. In Chuanren Ke (ed.), Routledge handbook of Chinese second language acquisition, 63-78. London: Routledge. https://doi.org/10.4324/9781315670706-4.

Johnson, David. \& Roger Johnson. 1975. Learning together and alone, cooperation, competition, and individualization. Englewood Cliffs, NJ: Prentice-Hall.

Ke, Chuanren \& Yan-Hui A. Li. 2011. Chinese as a foreign language in the U.S. Journal of Chinese Linguistics 39(1). 177-238.

Ke, C. \& H. H. Shen. 2003. Research and theory building in teaching Chinese in the U.S.: A comprehensive review and critique. Language Teaching and Linguistic Studies 94(3). 1-17.

Klette, Kirsti. 2011. The role of theory in educational research. In The role of theory in educational research: Report from the March seminar 2011, 3-7. Hanshaugen, Norway: The Research Council of Norway. Retrieved from: https://www.forskningsradet.no/siteassets/ publikasjoner/1253979441594.pdf.

Krashen, Stephen. 1985. The input hypothesis: Issues and implications. New York: Longman.

Krus, David J. 1977. Order analysis: An inferential model of dimensional analysis and scaling. Educational and Psychological Measurement 37(3). 587-601.

Kruschke, John K. \& Nathaniel J. Blair. 2000. Blocking and backward blocking involve learned inattention. Psychonomic Bulletin \& Review 7. 636-645.

LaBerge, David \& Jay Samuels. 1974. Toward a theory of automatic information processing in reading. Cognitive Psychology 6(2). 293-323.

Lantolf, James P. \& Steven L. Thorne. 2007. Sociocultural theory and second language acquisition. In Bill van Patten \& Jessica Williams (eds.), Theories in second language acquisition, 201224. Mahwah, NJ: Lawrence Erlbaum Associates, Inc.

Lantolf, James P. 2000. Sociocultural theory and second language learning. England: Oxford University Press.

Long, Michael H. 1996. The role of the linguistic environment in second language acquisition. In William C. Ritchies \& Tej K. Bhatia (eds.), Handbook of second language acquisition, 413468. San Diego, CA: Academic Press. https://doi.org/10.1016/b978-012589042-7/50015-3. 
Ma, Qing. 2014. A contextualized study of EFL learners'vocabulary learning approaches: Framework, learner type and degree of success. Asia TEFL 11(3). 33-71.

Mayer, Richard E. 2005. Cognitive theory of multimedia learning. In R. E. Mayer (ed.), The Cambridge handbook of multimedia learning, 31-48. New York, NY: Cambridge University Press. https://doi.org/10.1017/cbo9780511816819.004.

Mayer, Richard E. 2009. Multimedia learning, 2nd edn. New York, NY: Cambridge University Press. McDonough, Kim. 2006. Action research and the professional development of graduate teaching assistants. The Modern Language Journal 90(1). 33-47.

McLaren, Peter \& Nadia Jaramillo. 2007. Pedagogy and praxis in the age of empire: Towards a new humanism. Rotterdam: Sense Publishers.

McMillan, James H. \& Sally Schumacher. 2000. Research in education: A conceptual introduction. London: Longman.

Meng, Yanhua. 2017. Chinese language education in America: An analysis of the policy changes. Sinologia Hispanica, China Studies Review 5(2). 49-62.

Meyers, Chet \& Thomas B. Jones. 1993. Promoting active learning. San Francisco: Jossey-Bass Publishers.

Mu, Aili \& De Zhang. 2005. Teaching Chinese films with digitalized modules in WebCT. Journal of the Chinese Language Teachers Association 40(3). 29-48.

Nathan, Norman. 1966. A classroom innovation - Contracts and self-paced instruction in Chinese. Journal of the Chinese Language Teachers Association 1(2). 58-64.

Oxford, Rebecca. 1994. Language learning strategies: An update. ERIC Digest: ED376707.

Paivio, Allan. 1971. Imagery and verbal processes. New York: Holt Rinehart \& Winston.

Paivio, Allan. 1986. Mental representations: A dual-coding approach. New York: Oxford University Press.

Phinney, Marianne. 1996. Exploring the virtual world: Computers in the second language writing classroom. In Martha Pennington (ed.), The power of CALL, 137-152. Houston, TX: Athelstan.

Pieneman, Manfred. 1989. Is language teachable? Psycholinguistic experiments and hypotheses. Applied Linguistics 10(1). 52-79.

Potts, Diane. 2005. Pedagogy, purpose, and the second language learner in on-line communities. The Canadian Modern Language Review 62(1). 137-160.

Rabab'ah, Ghaleb. 2002. Second language communication strategies: Definitions, taxonomies, data elicitation methodology and teachability issues: A review article. ERIC Digest: ED472698.

Rovai, Alfred P. 2001. Building classroom community at a distance: A case study. Educational Technology Research \& Development 49(4). 33-48.

Schmidt, Richard. 1990. The role of consciousness in second language learning. Applied Linguistics 11(2). 206-226.

Schmidt, Richard. 1995. Consciousness and foreign language learning: A tutorial on the role of attention and awareness in learning. In Richard Schmidt (ed.), Attention and awareness in foreign language learning, 1-63. Honolulu: University of Hawaii Press.

Schmidt, Richard. 2001. Attention. In Peter Robinson (ed.), Cognition and second language acquisition, 3-32. Cambridge: Cambridge University Press. https://doi.org/10.1017/ cbo9781139524780.003.

Schwartz, Bonnie D. \& Rex A. Sprouse. 1996. L2 cognitive states and the full transfer/full access model. Second Language Research 12(1). 40-72.

Shi, Feng \& Baoying Wen. 2009. Overview of the second language acquisition of Chinese. Journal of Chinese Linguistics 37(1). 130-144. 
Suppes, Patrick. 1974. The place of theory in educational research. Educational Researcher 3. 3-10.

Swain, Merrill. 1995. Three functions of output in second language learning. In Guy Cook \& Barbara Seidhofer (eds.), Principles and practice in applied linguistics, 125-144. New York, NY: Oxford.

Sweller, John. 1988. Cognitive load during problem solving: Effects on learning. Cognitive Science 12(2). 257-285.

The New London Group. 2000. A pedagogy of multiliteracies: Designing social futures. In Bill Cope \& Mary Kalantzis (eds.), Multiliteracies: Literacy learning and the design of social futures, 9-39. Youth Yarra, Australia: MacMillan.

VanPatten, Bill. (ed.). 2004. Processing instruction: Theory, research, and commentary. Mahwah, NJ: Lawrence Erlbaum.

Vygotsky, Lev S. 1978. Mind in society: The development of higher psychological processes. Cambridge, MA: Harvard University Press.

Walker, Galal. 2000. Performed culture: Learning to participate in another culture. In Richard D. Lambert \& Elana Shohamy (eds.), Language and pedagogy: Essays in honor of A. Ronald Walton, 221-236. Amsterdam/Philadelphia: John Benjamins Publishing. https://doi. org/10.1075/2.96.14wal.

Wallace, Michael. 1998. Action research and teachers. Cambridge: Cambridge University Press. Waninge, Freerkien, Kees de Bot \& Zoltan Dornyei. 2014. Motivational dynamics in language learning: Change, stability, and context. The Modern Language Journal 98(3). 704-723.

Wen, Xiaohong. 2019. Research in second language acquisition of Chinese: An Introduction. In Xiaohong Wen \& Xin Jiang (eds.), Studies in learning and teaching Chinese as a second language, 1-18. New York: Routledge. https://doi.org/10.1007/978-981-15-0271-2_1.

Wittrock, Merlin C. 1974. Learning as a generative process. Educational Psychologist 11(2). 87-95. Yang, Chunsheng \& Han Luo. 2017. The applicability of SLA research to L2 pedagogy: Focusing on Mandarin tones. Second Language Learning Research 2(2). 62-79.

Yano, Yasukata, Michael H. Long \& Steven Ross. 1994. The effects of simplified and elaborated texts on foreign language reading comprehension. Language Learning 44(2). 189-219.

Yao, Tao-Chong \& Kuang-Tian Yao. 2010. Chinese language instruction in the United States: A look at its history and current status. In Hanhui Zhang (ed.), Chinese studies in North America: Research and resources, 773-784. Beijing: Zhonghua shu ju.

Yuan, Fangyuan \& Maiheng S. Dietrich. 2004. Formal instruction, grammatical teachability, and acquisition of Chinese as a second/foreign language. Journal of the Chinese Language Teachers Association 39(2). 1-18.

Yuan, Fangyuan. 2018. Roles of action research in the professional development of Chinese language teachers. Chinese as a Second Language 53(3). 201-221.

Zhang, Hang. 2014. The third tone: Allophones, sandhi rules and pedagogy. Journal of the Chinese Language Teachers Association 49(1). 117-45. Q4.

Zhang, Shenglan. 2017. Applying research-based multimedia design principles in designing and teaching beginning CFL learners Ba-construction online: A pilot study. Chinese as a Second Language 52(3). 255-291.

Zhang, Zheng-Sheng. 2014. Research as seen from the editor of JCLTA. In Paper presented at the National Chinese Language Conference, Los Angeles, CA.

Zhang, Zheng-Sheng. 2018. JCLTA has turned fifty! In Vivian Ling (ed.), Chinese language education in American academia in the 20th century: A collective retrospective, 202-212. New York: Routledge. 


\section{Bionote}

\section{Shenglan Zhang}

lowa State University, 3118 D Pearson Hall, Ames, IA 50011, USA

shenglan@iastate.edu

Shenglan Zhang is an associate professor in the Department of World Languages and Cultures at lowa State University in the United States, where she is Coordinator for the Multi-Section Lower Division Courses in the Chinese Studies Program. She earned her PhD in Educational Psychology and Educational Technology at Michigan State University. Her research interests include computer-assisted language learning (CALL), designing for blended, flipped, and online learning, language teaching pedagogy, and self-regulated learning. 